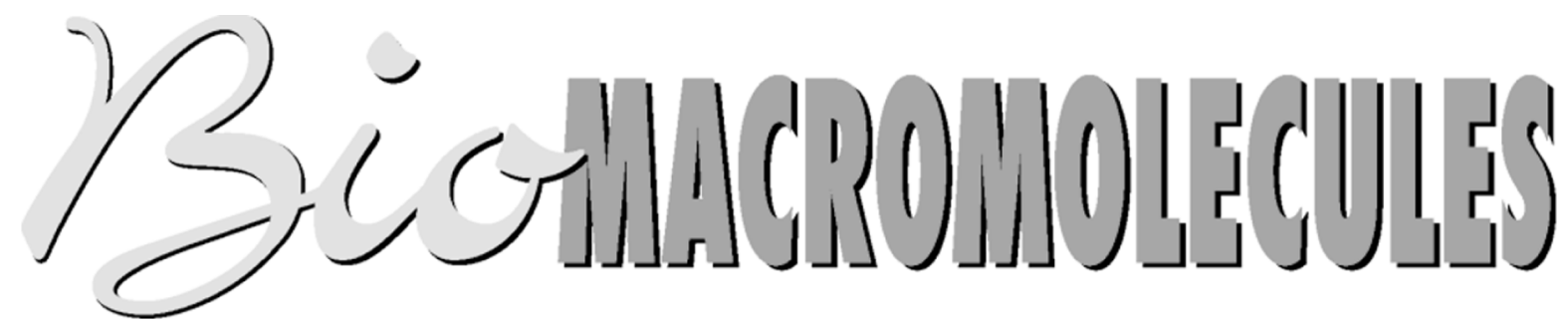

June 2009

\title{
Communications
}

\section{Controlling the Morphology in DNA Condensation and Precipitation}

\author{
Maria F. V. Pinto, ${ }^{\dagger, \ddagger}$ M. Carmen Morán, ${ }^{\dagger}$ M. Graça Miguel, ${ }^{\dagger}$ Björn Lindman, ${ }^{\dagger}$ \\ Amália S. Jurado, ${ }^{\ddagger}$ and Alberto A. C. C. Pais ${ }^{\star} \dagger$ \\ Chemistry Department, Coimbra University, Rua Larga, 3004-535 Coimbra, Portugal, and Biochemistry \\ Department, Coimbra University, Rua dos Estudos, 3001-401 Coimbra, Portugal
}

Received February 17, 2009; Revised Manuscript Received May 6, 2009

\begin{abstract}
This work addresses the influence of solution inhomogeneity on conformation, aggregation, and coil/globule and bundle/single chain coexistence of T4 DNA molecules. The inhomogeneity is induced by mixing two solutions containing, respectively, protamine and DNA, with different relative concentrations, but aiming at producing the same final concentrations. The study was conducted by means of fluorescence microscopy (FM), complemented with scanning electron microscopy (SEM). It is shown that the degree of precipitation, the structures formed, and the relative population of compacted and unfolded structures are highly dependent on the method of preparation of the mixtures that contain the DNA/protamine complexes. Most of the structures reported in the literature, that is, overcharged/undercharged globules, toroids, chains internally segregated, and bundles composed of several chains were observed in our different mixtures of fixed final concentration.
\end{abstract}

\section{Introduction}

Understanding the factors that govern the size and shape of DNA condensates is believed to be important for the optimization of gene delivery. ${ }^{1-3}$ In the living cellular environment, long DNA molecules are folded into a compact state to store, transport, and preserve the genetic material. ${ }^{4}$ However, it is wellknown that long DNA chains in aqueous solution adopt an elongated coil conformation. This contrasts with the folded state of DNA in living cells, and DNA condensation in vitro has been intensely studied as a model for DNA compaction in vivo. Double-helix DNA condensation includes two types of phenomena: single-chain folding and aggregation (precipitation) as a multichain process. ${ }^{5}$

It has been found that isolated DNA chains undergo a coil-globule transition upon increasing concentration of condensing agents in solution. Compaction of DNA chains is induced in vitro by the addition of agents such as basic proteins, polyamines, multivalent metal cations, hydrophilic polymers, cationic polymers, catanionic liposomes, and cationic and

\footnotetext{
* To whom correspondence should be addressed. E-mail: pais@qui.uc.pt.

Chemistry Department.

Biochemistry Department.
}

nonionic surfactants. ${ }^{6-11}$ In vitro studies use various techniques, such as dynamic light scattering, viscometry, electron and atomic force microscopy, fluorescence microscopy, and transmission electron microscopy to observe DNA morphologies.

The objective of the present study is to investigate the influence of solution inhomogeneity upon the final conformation, aggregation, and precipitation behavior of T4 DNA molecules by means of fluorescence microscopy, using protamine sulfate as condensing agent. Protamines are highly positively charged (overall charge +21 ) arginine-rich proteins that bind to DNA in a nonspecific manner via electrostatic interactions. In addition, protamine sulfate has been shown to condense DNA,${ }^{11,12}$ to form DNA gel particles for modulating DNA release in vitro ${ }^{13}$ and to deliver plasmid DNA into eukaryotic cells. ${ }^{14}$ The inhomogeneity is induced by mixing two solutions containing, respectively, protamine and DNA and organized in sets. These sets are prepared with different DNA/protamine volume ratios and range from concentrated DNA/diluted protamine sulfate to diluted DNA/concentrated protamine sulfate. The same final concentrations for both the nucleic acid and the cationic agent are obtained for all sets.

In the present work, protamine concentrations of 0.05 and $1.0 \mu \mathrm{M}$ were selected considering our previous work. ${ }^{11}$ The 


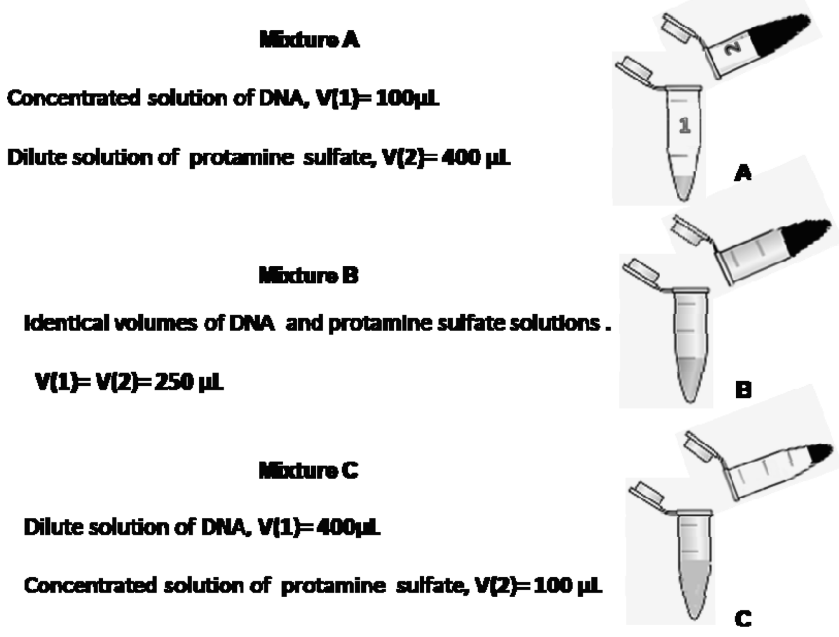

Figure 1. Scheme of experimental procedure describing the different sets and the respective DNA/protamine volume ratios.

influence of solution inhomogeneity was studied using a protamine concentration of $0.05 \mu \mathrm{M}$, which is situated in the range of concentrations for which coexistence between coils and globules is observed. The higher value corresponds to total conversion to globules. These have been assessed independently in what concerns the effect of the mixing volume ratio, and the results for each concentration were, in turn, compared. It was observed that the effect of the mixing ratio was not the same at different concentrations.

\section{Experimental Section}

Materials. Bacteriophage T4 DNA (165.6 kilobase pairs, contour length of $57 \mu \mathrm{m}$ ) was purchased from Wako Nippon Gene. The fluorescence dye 4,6-diamidino-2-phenylindole (DAPI; excitation/ emission $=360 / 460 \mathrm{~nm}$ ), the antioxidant, 2-mercaptoehanol (ME), and the buffer salt, Trizma base, were purchased from Sigma. Protamine sulfate salt from salmon, grade $\mathrm{X}$, with a molecular weight approximately $5.1 \mathrm{kDa}$ (major component), was purchased from Sigma and used as received. All experiments were performed using Millipore Milli-Q deionized water (18.2 M $\Omega / \mathrm{cm}$ resistivity).

Sample Preparation. Solutions were prepared as described in what follows. T4 DNA was diluted with $\mathrm{pH} 7.610 \mathrm{mM}$ Tris $\mathrm{HCl}$ buffer containing $4 \%$ (v/v) ME and DAPI $(0.25 \mu \mathrm{M})$. Protamine was diluted in the same buffer solution. Sets of two solutions of different volumes (respectively containing protamine and DNA) were mixed (see Figure 1) to produce the same final concentrations (DNA concentration was $0.25 \mu \mathrm{M}$ in terms of nucleotide units and protamine 0.05 or $1.0 \mu \mathrm{M}$ ). In all mixtures the final volume after mixing was $500 \mu \mathrm{L}$.

The mixing procedure consisted of pouring the protein solution over the solution containing DNA. Subsequently, and to achieve total mixing, the final solution was gently turned over twice. An aliquot $(7 \mu \mathrm{L})$ of each solution was removed from the tube, placed onto the glass microscope slide, covered with the coverslip, and sealed. It was immediately examined in the fluorescence microscope. The time between preparation and imaging of the solutions did not exceed 5-10 min. A longer equilibration would promote the deposition of some of the condensates, before transfer, thus biasing the sampling. In turn, a longer time of observation would decrease the quality of the images, if one takes into consideration parameters related to the fluorescence of the dye.

Fluorescence Microscopy. Samples were illuminated with a UVmercury lamp (100 W Ushio Olympus). The fluorescence images of DNA molecules were observed using a Olympus BX51 M microscope, equipped with a UplanFL N $100 \times / 1.30$ oil-immersed objective lens $(\infty / 0.17 / \mathrm{FN} 26.5)$ and a filter set type U-MNU2 (360-370 nm excitation and $400 \mathrm{~nm}$ dichromatic mirror). Images were digitized on a computer through a video camera (Olympus digital camera DP70) and were analyzed with an image processor (Olympus DP Controller 2.1.1.176, Olympus DP Manager 2.1.1.158). All observations were carried out at $25^{\circ} \mathrm{C}$. Special care was taken to thoroughly clean the glass microscope slides (Marienfeld) and coverslips with ethanol before each observation so as to prevent DNA degradation and precipitation onto the glass surface.

To evaluate changes in the shape and conformation of DNA molecules, representative images of all the different systems were recorded, mostly consisting of movies $7 \mathrm{~s}$ long (ca. 25 frames). Analyses of the images were performed using the public domain Image J, version $1.31 \mathrm{v}$ program (http://rsb.info.nih.gov/ij/). The relative populations in each sample were determined by counting the number of DNA molecules in each situation. At least 100 DNA condensates were counted for each sample. All the fluorescence microscopy images presented in this work were digitally enhanced.

Scanning Electron Microscopy. A scanning electron microscopy (Philips XL30- TMP) was used to evaluate the morphology of the different DNA condensates. Previously, the entire volume of the final solutions was frozen and lyophilized overnight immediately after mixing $\left(-46{ }^{\circ} \mathrm{C}, 0.035 \mathrm{mBar}\right)$. Subsequently, the dry samples were dispersed on a double-sided adhesive conductive tape attached to a SEM stub and underwent sputter-coating with gold. The following day, samples were transferred onto the microscope stage and examined at $5 \mathrm{kV}$.

\section{Results and Discussion}

It is a general finding that DNA molecules condense into different morphologies depending on solution conditions (e.g., ionic strength), DNA properties (e.g., contour length, persistence length), and the nature of the condensing agent. ${ }^{15}$ In this work, the use of different volume ratios for the preparation of the mixtures is examined in terms of the control not only of the DNA morphologies but also on the relative population of compact and less compact DNA.

Concentrated Protamine System $(1.0 \mu \mathrm{M})$. The influence of solution inhomogeneity was initially studied using a protamine concentration of $1.0 \mu \mathrm{M}$. As was indicated before, total conversion to globules occurs for this concentration. ${ }^{11}$

In this study, it was observed that for these conditions, most DNA molecules precipitate onto the glass, either as a single chain or in the form of multichain aggregates. This is consistent with previous results that have shown a tendency for DNAprotamine complexes to aggregate. ${ }^{16}$ Figure $2 \mathrm{a}$ shows the distribution of DNA both on the glass and in the bulk for the different mixtures. In spite of a predominance of precipitated complexes, it is seen that the number of DNA condensates in the bulk increases from A to C. FM observations further indicate that, in mixture A, a coexistence between single chain globules and rod-like and V-shaped aggregates is found (Figure 2b). This coexistence of bundle structures with multiple chains and compact states with single chains was already described in the literature. ${ }^{17}$ However, the experimental procedure involves changes in the DNA concentration. It is known that different structures can be found depending on solution conditions and condensing agent. Typically, single DNA molecules condense into toroids, rod-like shapes, or globules. ${ }^{18,19}$

Representative fluorescence microscopy images of the structures obtained in this study are shown in Figure 3. The existence of these morphologies was also confirmed by scanning electroscope microscopy, SEM (see Figure 4). We note that the use of this technique is not common for the study of DNA condensation. The present results indicate, however, that it may be an excellent tool to observe in detail the structures obtained 

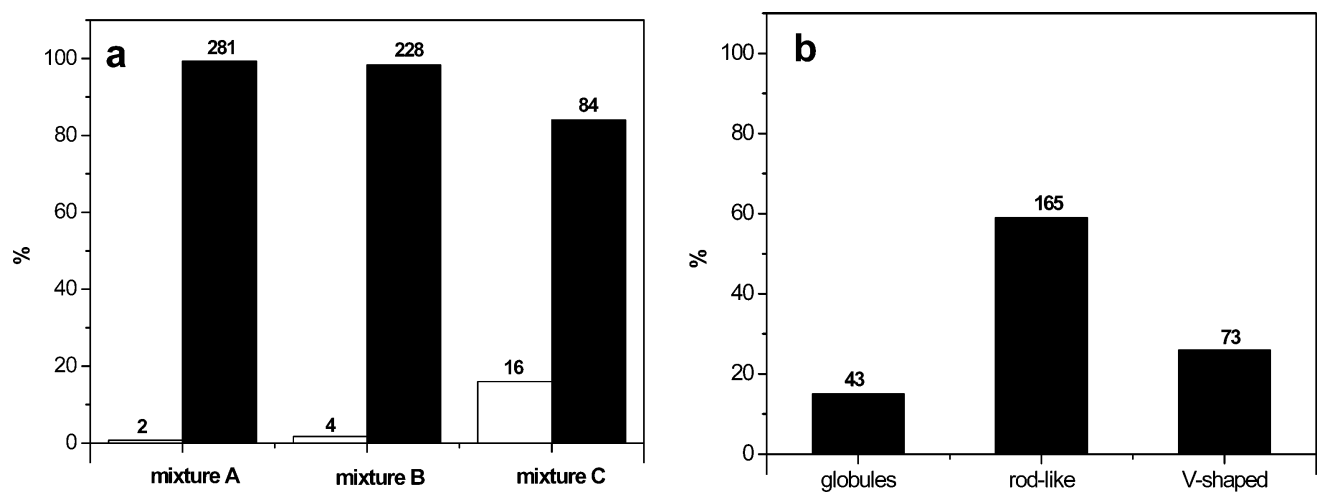

Figure 2. (a) Histogram of the population of DNA in bulk (white columns) and in the glass (black columns) for the three different mixtures of Figure 1. (b) Histogram of the population of the different DNA precipitates in the glass for mixture A. The number of DNA molecules considered in each situation is indicated on the top of the columns. Values correspond to a protamine concentration of $1.0 \mu \mathrm{M}$.
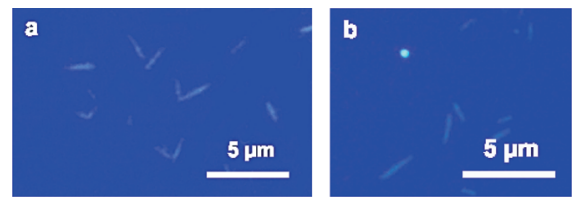

Figure 3. Fluorescence images of different T4 DNA condensate morphologies observed onto the glass for the mixture A: (a) rod-like and $\mathrm{V}$-shaped aggregates and (b) rod-like and globules condensates.
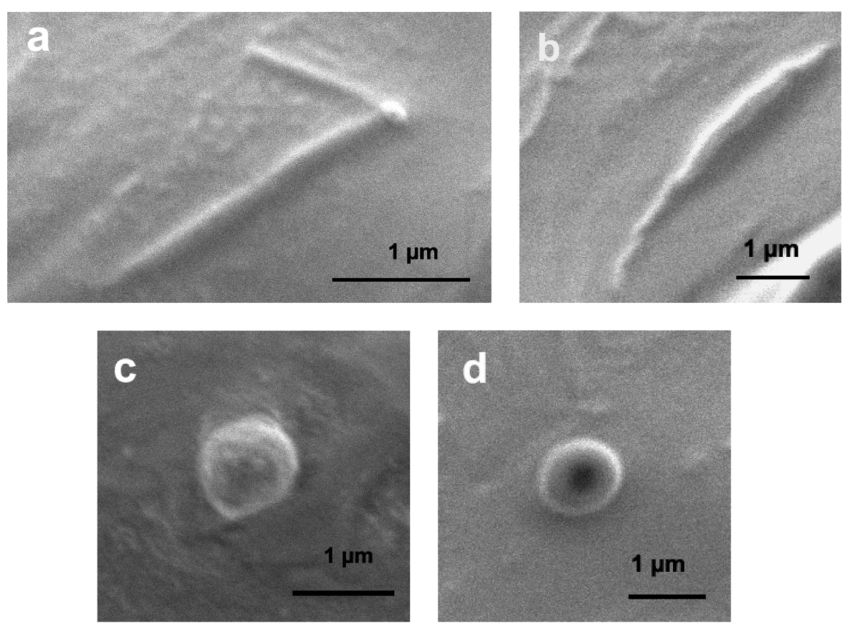

Figure 4. Scanning electron micrographs of the different T4 DNA morphologies condensates found for the mixture A: V-shaped (a), rod-like (b), globular (c), and toroidal (d).

in such studies. Moreover, it provides additional information on those structures. For instance, the occurrence of blurring makes it unlikely to identify toroidal shapes of low radii (typically of the order of $1.0 \mu \mathrm{m}$ ) resorting to FM observations. SEM has shown that toroids are also present (Figure 4d) with dimensions similar to those of globules.

For mixtures $\mathrm{B}$ and $\mathrm{C}$ the number of aggregates is negligible and globules dominate (results not shown). The morphology of DNA molecules found on the glass is essentially the same as that of those found in solution. However, the globules in solution are larger than those in the glass. For the case of mixture C, the only one in which the amount of globules in solution allows for a reliable estimate of size, it is observed that globules in solution are, on average, larger by about $23 \%(0.96 \mu \mathrm{m}$, when precipitated over the glass, and $1.17 \mu \mathrm{m}$ in solution). The soluble nature of the globules indicates that their surfaces are charged, which is considered to be responsible for the enlargement in solution. This probably results from the repulsion between positively charged amino groups in overcharged complexes. ${ }^{17}$ In contrast, the degree of neutralization in the precipitated globules is obviously estimated as close to $100 \%$.

One of the aspects worth being discussed is why multichain aggregates are present in mixture A and absent in the other two cases. For the former, DNA is initially concentrated in a smaller volume. There is a driving force for the separation of the respective chains, but if the cationic agent starts to bind before extensive separation, aggregates may be formed. In mixtures B and C, DNA molecules are initially scattered in a larger volume, and thus, the formation of multichain structures is less probable. Another aspect is the presence of rod-like and V-shaped structures. The former are essentially straight, while the others have only one region of bending between two straight portions. This is probably related with the competition between folding and bundling indicated in previous work. ${ }^{20}$ In fact, for multichain structures the aggregation may be side to side and more or less in a straight conformation so as to avoid the bending energy penalty.

Diluted Protamine System $(0.05 \mu \mathrm{M})$. Analogous studies were carried out using a lower protamine concentration $(0.05$ $\mu \mathrm{M})$. We recall that this value is situated in the range of concentrations for which coexistence between coils and globules is observed. ${ }^{11}$

For this diluted protamine system, it is seen that almost all DNA condensates are present in the bulk, with a complete absence of multichain aggregates. These observations result simply from the fact that the action of the lower concentration protamine is less effective and starts after DNA molecules diffuse and separate.

The structures found in bulk are globules and unfolded chains. The former are characterized by a very bright central part, the core that concentrates most of the dye, which remains unchanged during the whole period of observation. The average size of these globular structures is about $1.0 \mu \mathrm{m}$. The unfolded chains, with the longest axis in excess of $1.5 \mu \mathrm{m}$, cannot be regarded as random coils. They are mostly partially unfolded chains with some degree of intrachain segregation, indicating an incomplete process of compaction. The formation of these structures instead of the globular ones is due to the electrostatic repulsion of uncompensated charges of DNA. ${ }^{21}$

This coexistence behavior can be explained considering the difference in the diffusion coefficients of DNA and protamine molecules. In fact, DNA can be regarded as effectively stationary in relation to protamine. In these mixtures, protamine molecules, with a higher diffusion coefficient, are attracted to the region where DNA is present. The first DNA molecules that come in 


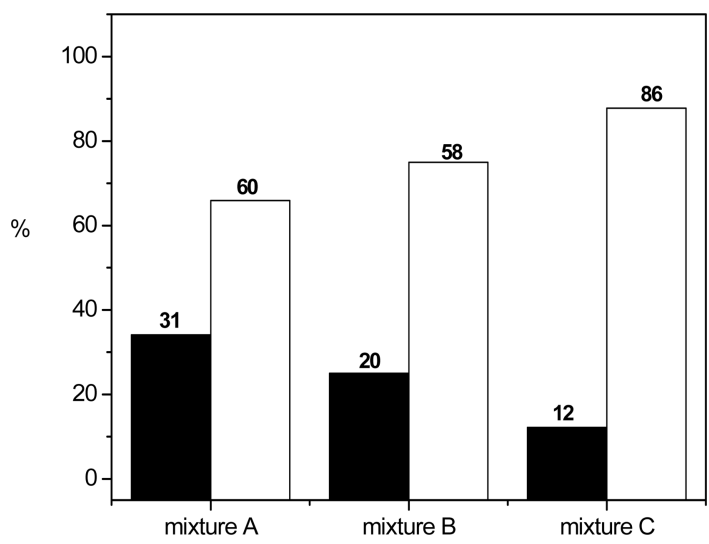

Figure 5. Distribution between DNA globules (black columns) and unfolded DNA chains (white columns) observed in the bulk for the three different mixtures. The number of DNA molecules observed for each situation is indicated on the top of the columns. Values correspond to a protamine concentration of $0.05 \mu \mathrm{M}$.

contact with protamine interact with a high number of these molecules, which is sufficient to promote the formation of globules. This reduces the amount of protamine available in other regions. The DNA molecules that are present further away from these initial mixing interfaces come in contact with a smaller number of protamine sulfate molecules, and compaction is, thus, only partial.

Figure 5 shows the distribution of the two types of structures. It is shown that for the concentrations under analysis, the unfolded chains are predominant in all the mixtures and that the relative amount of the two types of structures is strongly influenced by the mixing procedure. It is also shown that the fraction of globules decreases from mixture A to mixture C. In this order, DNA molecules become scattered in increasing volumes immediately after mixing. The more concentrated DNA, mixture A, consequently promotes a more significant inhomegeneity in the distribution of protamine, which is successively decreased in B and C. In other words, and using a more pictorial description, the systems behave as if DNA would act as a filter for protamine molecules. When DNA is initially more concentrated, the "openings" of the filter are smaller, and the retention of protamine in the initial contact regions is higher. This results in the formation of a higher number of globules and a lower number of partially unfolded molecules in the subsequent contact regions.

Figure 6 shows fluorescence microscopy images of the different T4 DNA structures observed on the bulk for a final protamine concentration of $0.05 \mu \mathrm{M}$. Apart from globules as the final product of DNA compaction (d), unfolded chains with one (a) or two $(b, c)$ globule-like units were also found.

\section{Conclusions}

The present work has shown that it is possible to obtain a variety of conformations, degrees of compaction and aggregation simply by manipulating the way DNA comes in contact with the condensing agent. Most of the structures reported in the literature, that is, overcharged/undercharged globules, toroids, chains internaly segregated, and bundles composed of several chains were observed in our different mixtures of fixed final concentration.

The results presented in this work suggest procedures to control the morphology of DNA condensates simply by changing volumes on the mixing protocol. We also propose SEM as
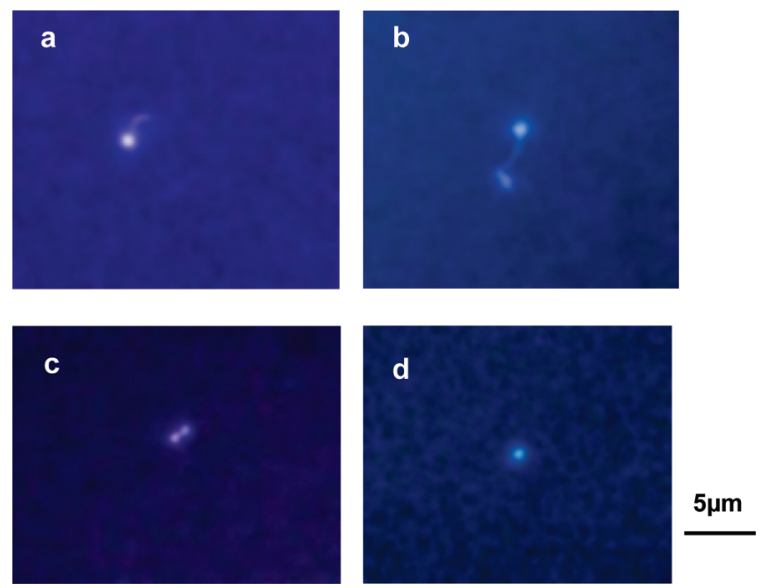

Figure 6. Fluorescence images of partially unfolded $(a-c)$ and compacted (d) T4 DNA observed in the bulk as induced by protamine at a concentration of $0.05 \mu \mathrm{M}$.

an effective technique to study the size and shape of the different morphologies.

We have specifically sketched a method (i) to control the number of globules in solution, (ii) for the production of bundles, (iii) to obtain overcharged globules, and (iv) for achieving an even distribution of the cationic agent over DNA. In summary, the number of globules in solution can be controlled by changing the DNA concentration prior to the mixing with relatively low concentration protamine solutions (from mixture A to $\mathrm{C}$; 0.05 $\mu \mathrm{M})$. Bundles are produced when concentrated DNA solutions are mixed into highly concentrated protamine solutions (mixture A; $1.0 \mu \mathrm{M})$. Overcharged globules result from relatively diluted DNA solutions combined with concentrated protamine solutions (mixture $\mathrm{C} ; 1.0 \mu \mathrm{M}$ ). Finally, even distribution is present when DNA and protamine are diluted (mixture $\mathrm{C} ; 0.05 \mu \mathrm{M}$ ) or when DNA and protamine, at relatively high concentrations (mixture $\mathrm{B} ; 1.0 \mu \mathrm{M}$ ), are mixed in comparatively large volumes. In the former situation, we obtain partially folded chains in solution, while in the latter the result is a homogeneous population of precipitated globules.

In future work, we plan to extend this study to other highly charged cationic agents and to an assessment of lifetimes and kinetic evolution of the structures. We plan to carry out experiments inducing a larger degree of inhomogeinity with further variations in the final concentrations. Other methods of mixing (e.g., resorting to rapid injections) will also be tested.

Acknowledgment. We acknowledge Prof. Amilcar Ramalho for making available the SEM equipment and help in obtaining the respective images. M.F.V.P. acknowledges FCT for Ph.D. Grant SFRH/BD/41793/2007. We also thank Fundação para a Ciência e Tecnologia (FCT), Portugal, for financial support under Project PTDC/QUI/67962/2006.

\section{References and Notes}

(1) Itaka, K.; Kataoka, K. Eur. J. Pharm. Biopharm. 2009, 71, 475-483.

(2) Thomas, M.; Klibanov, A. M. Appl. Microbiol. Biotechnol. 2003, 62, 27-34.

(3) Vijayanathan, V.; Thomas, T.; Thomas, T. J. Biochemistry 2002, 41, 14085-14094.

(4) Holde, K. E. Chromatin; Springer: Heidelberg, 1989.

(5) Iwaki, T.; Saito, T.; Yoshikawa, K. Colloids Surf., B 2007, 56, 126133.

(6) Eric, R.; Pelta, J.; Frutos, M. d.; Livolant, F. Phys. Rev. Lett. 2006, 97, 068103 .

(7) Bloomfield, V. A. Curr. Opin. Struct. Biol. 1996, 6, 334-341. 
(8) Dias, R. S.; Pais, A. A. C. C.; Miguel, M. G.; Lindman, B. J. Chem. Phys. 2003, 119, 8150-8157.

(9) Dias, R. S.; Pais, A. A. C. C.; Miguel, M. G.; Lindman, B. J. Chin. Chem. Soc. 2004, 447-469.

(10) Dias, R. S.; Pais, A. A. C. C.; Miguel, M. G.; Lindman, B. Colloids Surf., A 2004, 250, 115-131.

(11) Gaweda, S.; Morán, M. C.; Pais, A. A. C. C.; Dias, R. S.; Schillén, K.; Lindman, B.; Miguel, M. G. J. Colloid Interface Sci. 2008, 323, $75-83$.

(12) Brewer, L.; Corzett, M.; Balhorn, R. J. Biol. Chem. 2002, 277, 38895 38900.

(13) Moŕan, M. C.; Pais, A. A. C. C.; Ramalho, A.; Miguel, M. G.; Lindman, B. Langmuir 2009, submitted for publication.

(14) Saito, G.; Amidon, G. L.; Lee, K. D. Gene Ther. 2003, 10, 72-83.

(15) Vilfan, I. D.; Conwell, C. C.; Sarkar, T.; Hud, N. V. Biochemistry 2006, 45, 8174-8183.
(16) Allen, M. J.; Bradbury, E. M.; Balhorn, R. Nucleic Acids Res. 1997, $25,2221-2226$

(17) Toshio, I.; Satoru, K.; Takahiro, S.; Kenichi, Y.; Sergey, S. A. J. Chem. Phys. 2004, 120, 4004-4011.

(18) Raspaud, E.; Livolant, F.; Radler, J. Interaction of DNA with Cationic Polymers. In DNA interaction with polymers and surfactants; Rita, S, Dias, B. L., Eds.; John Wiley \& Sons, Inc.: Hoboken, New Jersey, 2008; pp 119-134.

(19) Balhorn, R. Gen. Biol. 2007, 8, 227.

(20) Koping-Hoggard, M.; Mel'nikova, Y. S.; Varum, K. M.; Lindman, B.; Artursson, P. J. Gene Med. 2003, 5, 130-14.

(21) Philippova, O. E.; Akitaya, T.; Mullagaliev, I. R.; Khokhlov, A. R.; Yoshikawa, K. Macromolecules 2005, 38, 9359-9365.

BM900211J 